\title{
Powerful Quasi-Steady-State Plasma Accelerator for Fusion Experiments
}

\author{
V.I. Tereshin ${ }^{1}$, V.V. Chebotarev ${ }^{1}$, D.G. Solyakov ${ }^{1}$, I.E. Garkusha ${ }^{1}$, V.A. Makhlaj ${ }^{1}$, \\ S.A. Trubchaninov ${ }^{1}$, N.I. Mitina ${ }^{1}$, A.I. Morozov ${ }^{2}$, A.V. Tsarenko ${ }^{1}$, and H. Wuerz ${ }^{3}$ \\ ${ }^{1}$ Institute of Plasma Physics of the National Science Center "Kharkov Institute of Physics and Technology", \\ 61108 Kharkov, Akademicheskaya St.,1, Ukraine \\ ${ }^{2}$ Russian National Center "Kurchatov Institute", Moscow, Russia \\ ${ }^{3}$ Forschugszenrum, Karlsruhe, IHM, Postfach 3640, D-76021, Karlsruhe, Germany
}

Received on 26 June, 2001

\begin{abstract}
The full-block quasi-steady-state plasma accelerator (QSPA) device and its operation as well as some possible applications of powerful plasma streams, generated by the QSPA, are described in this paper. The main attention was paid to plasma parameters measurements in the different regimes of operation. Analysis of efficiency of powerful plasma streams penetration into mirror magnetic field and plasma magnetization taking place under their propagation along the uniform magnetic field is carried out. Some results of thermal quench disruption simulations carried out with irradiation of solid targets by high energy plasma streams are presented in this paper.
\end{abstract}

\section{Introduction}

Different types of plasma accelerators were developed in previous time for plasma injection into magnetic traps (mainly for open-ended magnetic systems). Most of such accelerators of plasma were short pulse plasma guns of different geometry. Some experiments dealing with magnetic traps filled by plasma produced with such accelerators were also carried out. Nevertheless, due to the short pulse of the plasma streams (3-8 $\mu \mathrm{s})$ and limited ultimate level of stored energy in the plasma $(<100 \mathrm{~kJ})$ the interest of such experiments was relaxed at the last years. On the other hand, since the sixties, the quasi-steady-state plasma accelerators were being developed by A.I. Morozov [1] as a high power plasma source for fusion applications (in principle, the accelerators of such a type can generate plasma streams with energy more than several MJ and pulse durations of more than 1-10 ms). However, the experiments in simplified QSPA with non-transparent coaxial electrodes, investigated up to the end of the seventies, revealed some undesirable effects (like "anode current creep") resulting in disturbance of the accelerating process [2]. All these effects, restricting the plasma parameters to be achieved, were avoided in a two-stage accelerator with semi- transparent active or passive electrode-transformers [3-5]. One of the most powerful full- block QSPA (QSPA Kh-50) was installed in the IPP NSC KIPT.

The main aim of this work is to further investigate the QSPA operational regimes for generation of plasma streams with high plasma parameters and utilization of such plasmas for some fusion tasks. Plasma stream - magnetic field interaction is one of the fundamental problems of plasma physics, which is of importance for hot plasma injection into mirror magnetic traps of different kinds. This is present-day problem since plasma sources [5] with energy content in plasma of the order of $1 \mathrm{MJ}$ and with potential capability to produce plasma streams with energy up to 10-100 MJ appeared recently.

Magnetized plasma streams were also utilized in experiments for modelling situations on the divertor plate surfaces under the current disruptions [6]. Shielding plasma layers, appeared under irradiation of samples of different materials with plasma streams, and erosion and melt properties of these materials were investigated.

\section{Installation and plasma pa- rameters}

Experiments were carried out in the QSPA Kh-50 device [5]. The full-block powerful quasi-steady-state plasma accelerator consists of two stages. The first one is for plasma production and pre-acceleration. The second stage (main accelerating channel) is a coaxial system of shaped active electrodes-transformers with magnetically screened elements (those elements are current supplied either from independent power sources or branching partly the discharge current in self-consistent regime of operation). The first stage is the drift chan- 
nel in which plasma, generated with five input ionization chambers (IIC), is shuffled and flows into the second stage. IIC's are low-power coaxial accelerators of plasma with outer anode being $8 \mathrm{~cm}$ in diameter. The magnetic system of the QSPA Kh-50 anode transformer is a ten-pole three-row set of longitudinal rods circuited by consecutive row of low-resistance wires to maintain the magnetic field falling down along the channel (pie rods). The electrical currents flowing in the pie rods, as well as the discharge current flowing in the anode collector and in the cathode transformer components, form the magnetic emitting surface (MES - lower picture of Fig. 1)) with an average diameter of $50 \mathrm{~cm}$, a length of $80 \mathrm{~cm}$. The MES comprises ten zero magnetic field lines stretched along the $T_{A}$. Along these lines cold plasma propagates. Cold plasma is produced by anode ionization chambers (AIC). The ions going off the MES to the channel sustain the current transport process.

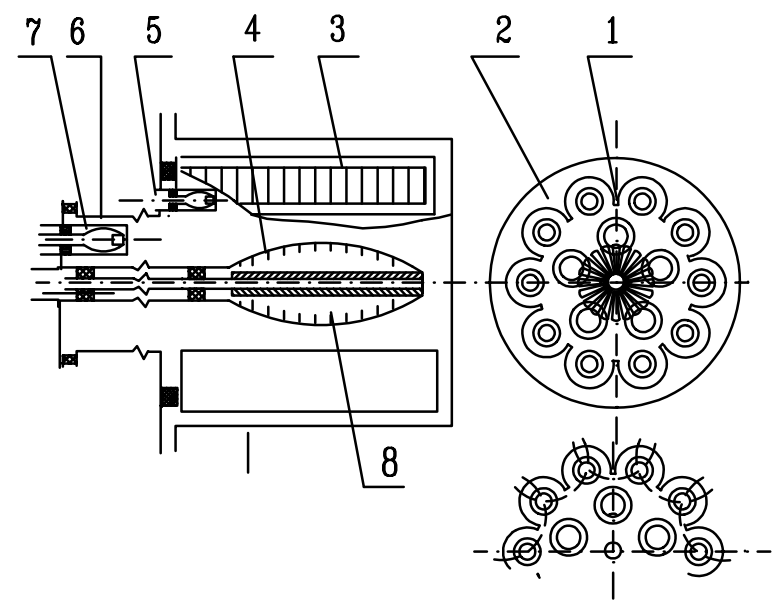

Figure 1. The block diagram of the QSPA Kh-50 device. 1: anode transformer; 2: anode collector; 3 : anode transformer pies; 4: cathode transformer rods; 5 : anode ionization chamber; 6: drift channel; 7: input ionization chambers; 8: needle- type emitters.

The tailored rod-like (20 rods) working part of the semiactive cathode $\left(T_{C}\right)$ has $62 \mathrm{~cm}$ in length, the largest diameter in the critical cross section being $36 \mathrm{~cm}$. Inside the hollow rods there are the current-carrying conductors, which form the magnetic system of the $T_{C}$. The discharge current takes its path to the $T_{C}$ rods via the needle-type cathodes fixed on the inner surface of the rods. Some experiments were carried out with cathodes of different types. The shaped surface was formed with 20 solid strips of $20 \mathrm{~mm}$ in width and $5 \mathrm{~mm}$ in thickness. Its diameter in critical cross-section achieved 40 $\mathrm{cm}$.

The working gas is hydrogen. The power supply of all accelerator units comes from capacitor banks. The total maximum energy of capacitors supplying the main discharge $W_{C}$ is about $2.25 \mathrm{MJ}$ (plus $2 \mathrm{MJ}$ for auxiliary systems of the QSPA). The main results were obtained with capacitor voltage of the main discharge up to 15 $\mathrm{kV}\left(W_{C} \approx 0.8 \mathrm{MJ}\right)$. The maximum discharge voltage achieved $12 \mathrm{kV}$ and maximum discharge current - 750 $\mathrm{kA}$.

The plasma streams were injected into a vacuum chamber of $1.5 \mathrm{~m}$ in diameter and $10 \mathrm{~m}$ in length. A magnetic solenoid of $1.6 \mathrm{~m}$ in length and $0.44 \mathrm{~m}$ in inner diameter (it consists of 4 separate magnetic coils) was placed at distance $z_{S}=1.25 \mathrm{~m}$ from accelerator output (closer to the compression region of a plasma stream) or $z_{S}=2.45 \mathrm{~m}$, where the incident plasma stream is diverging. Thus, the solenoid position defines the efficiency of plasma penetration into magnetic field. Samples of different materials to be irradiated by plasma were placed in a sluice chamber inside the diagnostic chamber. A vacuum chamber of $0.42 \mathrm{~m}$ in diameter, inserted in the magnetic solenoid, was joined with the two sections of the main vacuum chamber by using additional conical input and output adapter chambers. The maximum value of the magnetic field at the vicinity of the diagnostic chamber (between 3 -d and 4-th coils) achieved 1 T. Samples of different materials to be irradiated by plasma were placed in the sluice chamber in the diagnostic chamber.

Different regimes of plasma accelerator operation were utilized in these experiments. The first regime, short pulsed regime of operation, was realized when the main discharge was supplied by the whole condenser bank discharge, and current duration achieved $300 \mu \mathrm{s}$ (Fig. 2). To increase the discharge duration and, therefore, to increase the quasi-stationary phase of acceleration, the main condenser bank was composed of 6 sections switched on with some delay to each other - the second regime. This resulted in the increase an current discharge pulse (as well as plasma potential) up to 500 $\mu$ s (Fig. 2). The third regime was realized with simplified cathode transformer (operating in self-consistent regime) and non-sectioned condenser bank. Its integral characteristics are similar to the first regime, but plasma parameters are sufficiently different.

Plasma stream parameters, measured for the 1-st regime of operation at the distance of $1.5 \mathrm{~m}$ from the accelerator output (no magnetic field, vacuum chamber of $1.5 \mathrm{~m}$ ), were as follows. Plasma stream density was about $10^{15} \mathrm{~cm}^{-3}$, proton energy $200-450 \mathrm{eV}$, maximum power density achieved $20 \mathrm{MW} / \mathrm{cm}^{2}$, pulse duration (quasi-stationary phase) - 0.15-0.20 ms, total energy containment in plasma stream was about 0.4 MJ. The quasi-stationary phase of pulse duration at the 2-nd operational regime increased up to $0.4 \mathrm{~ms}$, but the average electron density and total plasma energy decreased. In the third regime of operation the plasma stream density was substantially increased (due to better plasma focusing at the accelerator output) and power pulse was decreased with respect to the first regime. 

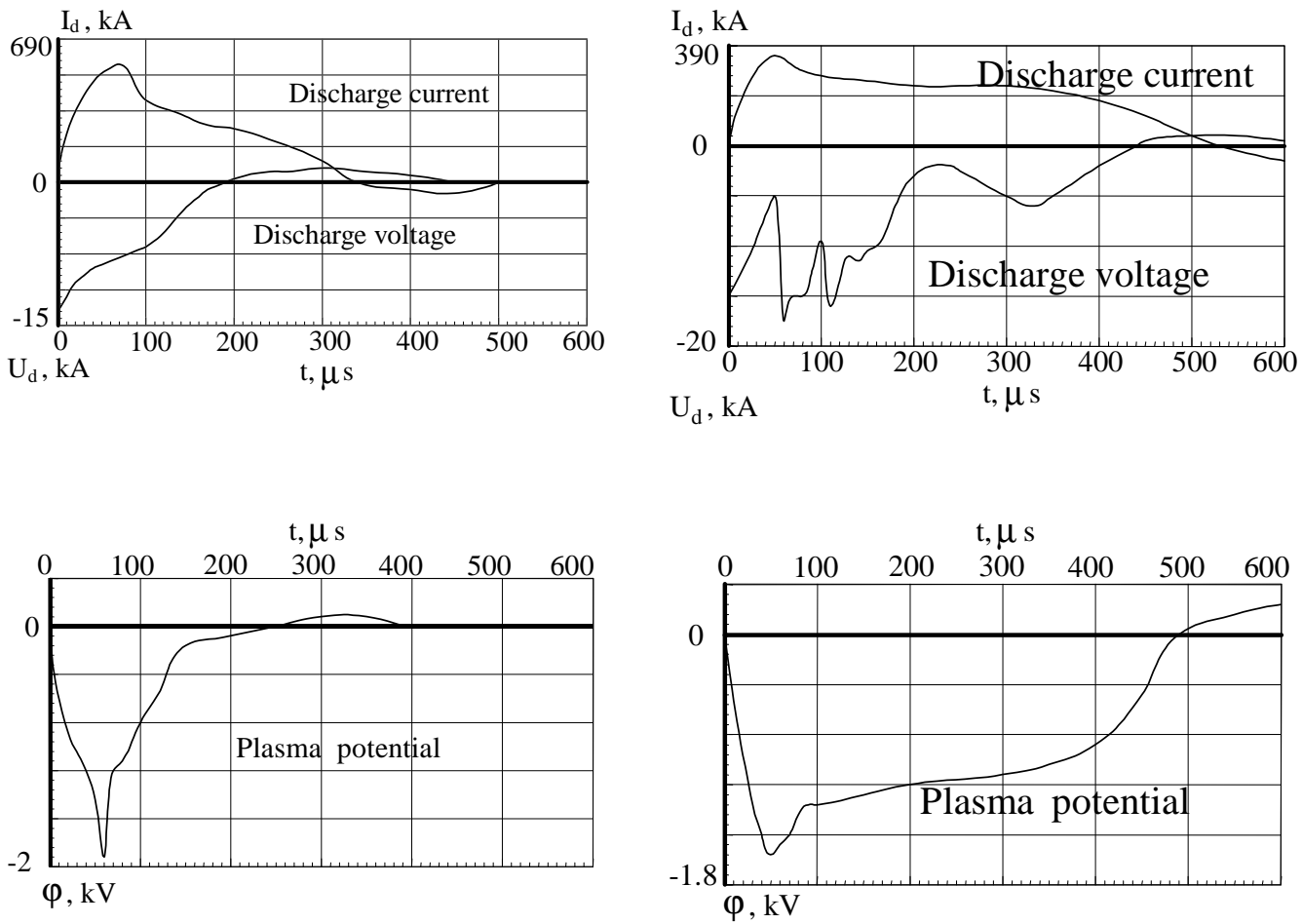

A

Figure 2. Signals of discharge current and voltage, and plasma potential: A - switching on the whole bank of capacitors; B programming switching on separate condenser banks.

\section{Plasma injection into mag- netic field}

The stray magnetic field of the solenoid at the vicinity of the accelerator is less than $1 \%$ of its maximum value. So, when speaking about plasma stream injection into magnetic mirror we have to analyze the non-magnetized plasma entering into a strong magnetic field, and its further magnetization. The efficiency of plasma penetration into the magnetic field has to depend on the magnetic field value, plasma stream parameters and its divergence just at the input to the magnetic mirror (operation regimes).

Time behavior of some plasma parameters, obtained in diagnostic chamber, with magnetic field $B_{Z}=0.72$ $\mathrm{T}$, for all operational regimes are shown in Fig. 3 .

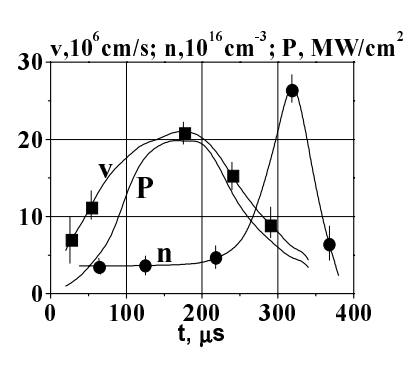

A

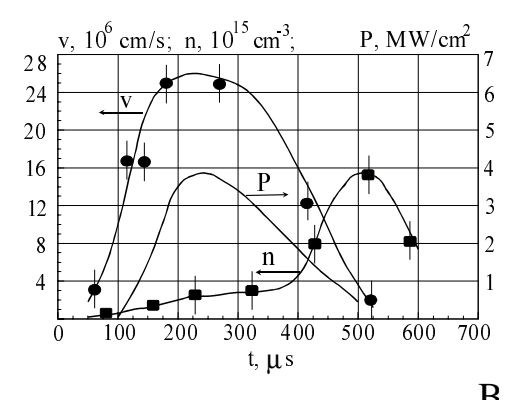

B

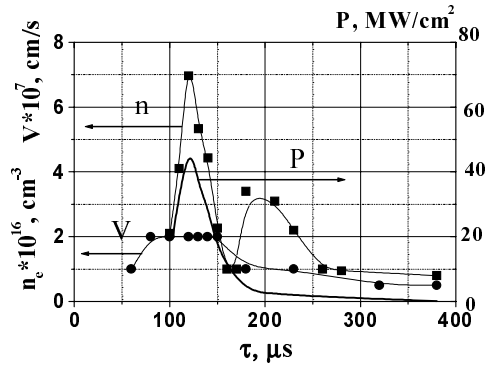

C

Figure 3. Time dependencies of plasma stream parameters in magnetic field $\left(B_{Z}=0.72 \mathrm{~T}\right)$ : velocity (v), electron density $(\mathrm{n})$ and power $(\mathrm{P})$; A - 1-st regime of the QSPA operation, short pulsed; B - 2-nd regime; C - 3-rd regime. 
Plasma density was deduced from Stark broadening measurements of $H_{\beta}$ spectral line, plasma velocity - by time of flight plasma between two magnetic probes, and plasma power was calculated on the base of density and velocity measurements. It follows from this picture that the power pulse is sufficiently longer for the 2-nd regime of operation, as compared to the 1st regime, and its duration (half width) achieves 0.23 $0.25 \mathrm{~ms}$. The values of power density for the long pulse regime are several times lower in comparison with short pulse regime. Since plasma velocities for both regimes are more or less comparable, this is mainly due to the decrease of plasma density in the 2-nd regime.

Plasma parameters measured for the 1-st regime at the diagnostic chamber with magnetic field strength of $0.54 \mathrm{~T}(z=2.35 \mathrm{~m})$ were as follows: plasma stream density was up to (2- 4).10 ${ }^{16} \mathrm{~cm}^{-3}$, proton energy achieved $200 \mathrm{eV}$, maximum power density was up to 20 $\mathrm{MW} / \mathrm{cm}^{2}$, electron temperature $2-3 \mathrm{eV}$, the duration of quasi-stationary phase of acceleration achieved 0.15 $0.17 \mathrm{~ms}$, total energy containment in plasma stream was about 0.1 MJ. The main plasma parameters for different operational regimes, distances from the accelerator output and magnetic field value are collected and presented in Table 1.

Analysis of energy containment in plasma streams passing through the magnetic solenoid, measured by the local calorimeters, showed that its absolute value depend strongly on magnetic solenoid position and mag- netic field value. Being $40-60 \%$ of the incident plasma stream energy for closer position of solenoid (for $B_{Z}=$ $0.36 \mathrm{~T}$ ), it falls down to about $25 \%$ for $B_{Z}=0.72 \mathrm{~T}$ and down to $5 \%$ for $z_{S}=3.55 \mathrm{~m}$. This is due to the higher plasma stream divergence just at the input to the solenoid and, therefore, more strong plasma reflection by the mirror in the latter case. At the third regime plasma energy content consists of about $25 \%$ but with higher electron density and energy density as compared to the first one.

The radial profiles of magnetic field in plasma normalized to vacuum field values, measured at the different cross-sections along the solenoid, including diagnostic chamber, for the first regime of QSPA operation, are shown in Fig. 4. One can see from these figures that plasma injection into magnetic field is accompanied by strong magnetic field displacement out of plasma at the input to the magnetic solenoid. Then, with plasma propagation along the solenoid, magnetic field penetrates into plasma. The efficiency of magnetic field penetration into plasma is in dependence of incident plasma parameters and magnetic solenoid position. Plasma radius, estimated as a plasma boundary where $B_{p l}=B_{0}$, decreases with the distance.

Table 1. Plasma streams characteristics at the diagnostic chamber (at the magnetic solenoid output).

\begin{tabular}{|c|c|c|c|c|c|}
\hline \multirow{3}{*}{$\begin{array}{c}\text { Plasma streams } \\
\text { parameters }\end{array}$} & \multicolumn{2}{|c|}{ 1-st regime - short pulse } & \multirow{2}{*}{\multicolumn{2}{|c|}{$\begin{array}{l}\text { 2-nd regime - long pulse } \\
\qquad \mathrm{z}=2.35 \mathrm{~m}\end{array}$}} & \multirow{3}{*}{$\begin{array}{c}\begin{array}{c}\text { 3-rd } \\
\text { regime }\end{array} \\
\mathrm{z}=2.35 \mathrm{~m} \\
\mathrm{~B}_{\mathrm{z} 0}=0.72 \\
\mathrm{~T}\end{array}$} \\
\hline & \multirow{2}{*}{\begin{tabular}{|c|}
$\mathrm{z}=2.35 \mathrm{~m}$ \\
$\mathrm{~B}_{\mathrm{z} 0}=0.54 \mathrm{~T}$
\end{tabular}} & \multirow{2}{*}{$\begin{aligned} \mathrm{z} & =3.55 \mathrm{~m} \\
\mathrm{~B}_{\mathrm{z} 0} & =0.72 \mathrm{~T}\end{aligned}$} & & & \\
\hline & & & $\mathrm{B}_{\mathrm{z} 0}=0.72 \mathrm{~T}$ & $\mathrm{~B}_{\mathrm{z} 0}=0.36 \mathrm{~T}$ & \\
\hline $\begin{array}{l}\text { Plasma density, } \\
\mathrm{n}_{\mathrm{e}}, \mathrm{cm}^{-3}\end{array}$ & $(2-4) \cdot 10^{16}$ & $(3-4) \cdot 10^{15}$ & $(2-3) \cdot 10^{15}$ & $(6-8) \cdot 10^{15}$ & $\begin{array}{c}(8- \\
10) .10^{16}\end{array}$ \\
\hline $\begin{array}{l}\text { Maximum plasma } \\
\text { velocity, } \mathrm{v}, \mathrm{cm} / \mathrm{s}\end{array}$ & $2.1 .10^{7}$ & $2.10^{7}$ & $2.6 .10^{7}$ & $2.5 \cdot 10^{7}$ & $2.1 .10^{7}$ \\
\hline $\begin{array}{l}\text { Total plasma } \\
\text { stream energy, } \\
\mathrm{W}, \mathrm{kJ}\end{array}$ & 98 & 21 & 15 & 25 & 92 \\
\hline $\begin{array}{l}\text { Maximum plasma } \\
\text { power, } \mathrm{P}, \mathrm{MW} / \mathrm{cm}^{2}\end{array}$ & 20 & 1.8 & 3.7 & 8 & 40 \\
\hline $\begin{array}{l}\text { Discharge duration, } \\
\mathrm{t}, \mathrm{ms}\end{array}$ & 0.36 & $0 . \overline{36}$ & 0.55 & 0.55 & 0.3 \\
\hline $\begin{array}{l}\text { Power pulse } \\
\text { duration, } \mathrm{t}, \mathrm{ms}\end{array}$ & $0.15-0.17$ & $0.03-0.05$ & $0.23-0.25$ & $0.2-0.22$ & $0.04-0.05$ \\
\hline Average $\beta$ & $0.15-0.2$ & $0.1-0.2$ & $0.1-0.15$ & $0.2-0.25$ & $0.5-0.6$ \\
\hline
\end{tabular}


Despite sufficiently different plasma parameters for different regimes of operation, it was found that the plasma stream radii are more or less comparable and equal $6-8 \mathrm{~cm}$ at the position of diagnostic chamber. As to the average $\beta$ value $\left(\beta=8 \pi n k T / B_{p l}^{2}\right)$, it is about $30-40 \%$ for the 1 -st operation regime, with essentially higher level of density, and 10-20\% for the 2-nd regime, indicating more effective plasma magnetization in the latter case. The average $\beta$ values estimated in different conditions of experiment are shown in Table 1.

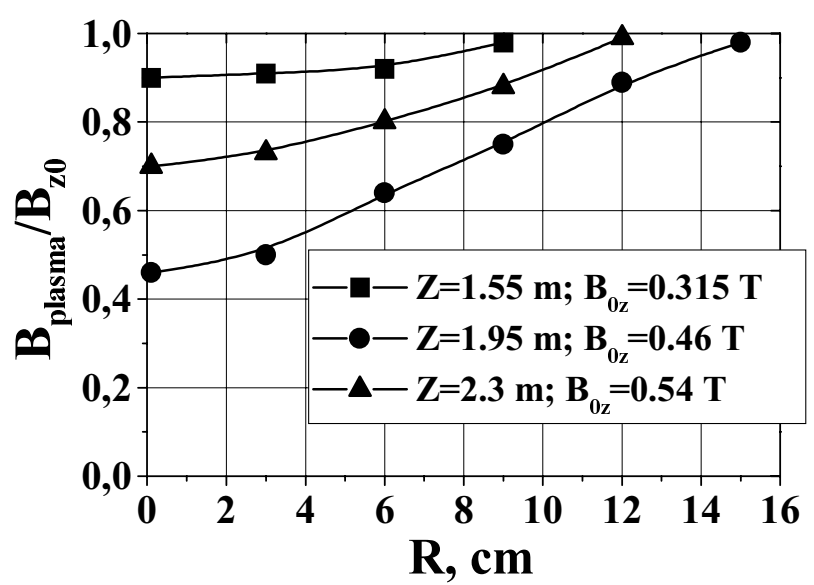

Figure 4. Radial distributions of magnetic field in plasma streams normalized to vacuum magnetic field values for the first operation regime.

\section{Plasma streams interaction with solid targets}

These experiments are for modelling the situation that can occur on the divertor plates of a tokamak-reactor under a thermal quench disruption. It was shown that under the incidence of powerful plasma streams to the target (graphite in this case) the shielding layer appeared close to the surface. Its density is more than one order of magnitude higher as compared to the density of incident plasma. Examples of density distributions in shielding layer are shown in Fig. 5. One can see that switching on the magnetic field leads to shielding layer thickness increase. The thickness $1-2 \mathrm{~cm}$ for sample irradiation with no magnetic field was increased up to $>$ $5 \mathrm{~cm}$ for $B_{z 0}=0.72 \mathrm{~T}$. This result can be explained by both material erosion and ionization and more effective stopping and confinement of plasma in the magnetic system with the end closed by material "mirror".

In the case of inclined surface irradiation the radial distribution of density is non-symmetric. The thickness of the shielding layer is the smallest at the upper edge of the sample (put forward to the plasma stream) and its value decreases with magnetic field switching on. For the central region of target (no magnetic field) the shielding layer density is 5 times lower for inclined plasma irradiation than for normal one. Density values are comparable for both normal and inclined irradiation in magnetic field of $0.72 \mathrm{~T}$. These layers are responsible for essential decreasing the part of incident plasma energy delivered to the surface and, therefore, the surface erosion.

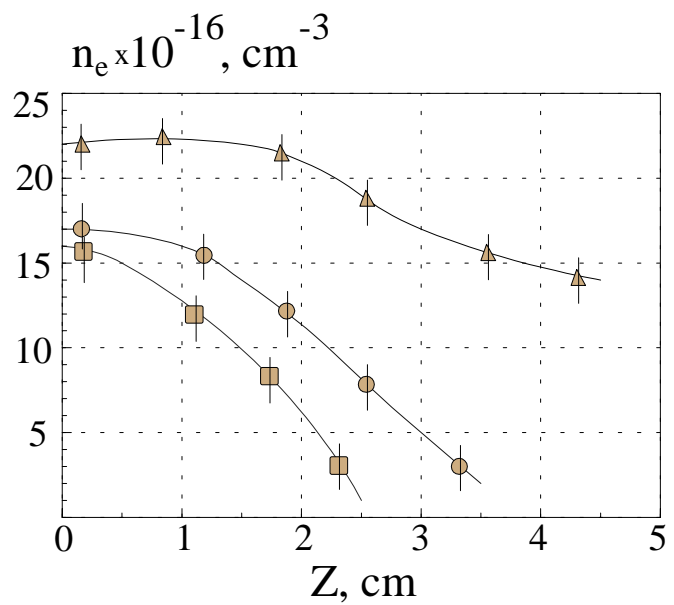

Figure 5. Plasma density in dependence of distance from the graphite target surface. $\left(\mathrm{P}=20 \mathrm{MW} / \mathrm{cm}^{2}\right) \Delta \tau=20 \mu \mathrm{s}$ from the beginning of a plasma interaction with the surface. $\boldsymbol{\square}-B_{z 0}=0 ; \bullet-B_{z 0}=0.36 \mathrm{~T} ; \boldsymbol{\Delta}-B_{z 0}=0.72 \mathrm{~T}$.

One of the erosion profiles of the graphite (MPG-7) target irradiated by plasma streams is shown in Fig. 6 . It was shown in our experiments [7] that the depth of graphite erosion is in strict dependence on the magnetic field. The erosion coefficient for normal plasma irradiation with $B_{z}=0$ was $2-3 \mu \mathrm{m}$ per $1 \mathrm{~kJ} / \mathrm{cm}^{2}$ of incident plasma $\left(P=20 \mathrm{MW} / \mathrm{cm}^{2}\right.$, power pulse length $0.17-0.2$ $\mathrm{ms})$. A much lower value of this coefficient $(0.3-0.4 \mu \mathrm{m}$ per $1 \mathrm{~kJ} / \mathrm{cm}^{2}$ ) was obtained for irradiation in a magnetic field of $0.72 \mathrm{~T}$. It increases at least by $1.5-2$ times for inclined target irradiation in magnetic field.

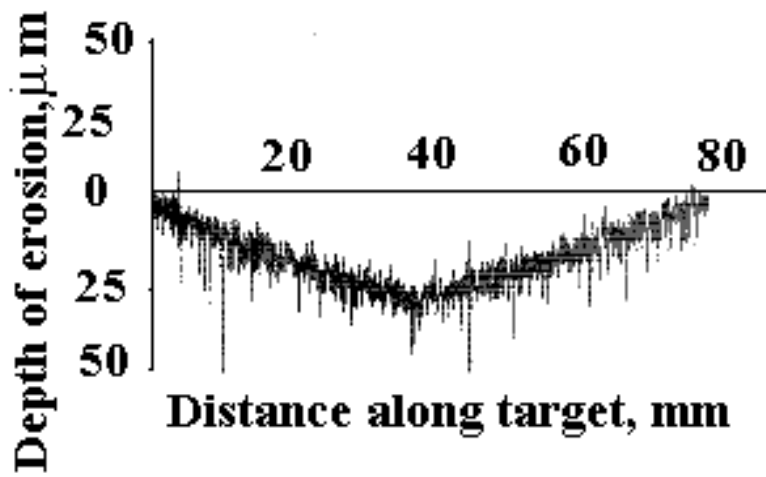

Figure 6. The profile of graphite surface erosion. 
The fine structure of the surface irradiated by powerful plasma was analyzed using quartz plates. Erosion profiles were measured after surface irradiation by one plasma shot $\left(P=20 \mathrm{MW} / \mathrm{cm}^{2}\right.$, pulse duration 0.15 $0.17 \mathrm{~ms})$ in a magnetic field of $0.7 \mathrm{~T}$. The plates $(9 \times 9$ $\mathrm{cm}$ in size) were irradiated by plasma both normally to the surface and under the angle of inclination $20^{\circ}$ with respect to the axis. For inclined irradiation, erosion profiles are shown in Fig. 7. The profiles are plotted for three sample cross-sections: median one and \pm $20 \mathrm{~mm}$ apart of it. The maximum values of erosion depth are comparable for both cases. Nevertheless, for inclined irradiation the erosion profiles show more complicated structure along the surface. They have periodical structure with periodicity of $5-6 \mathrm{~cm}$. This result is in agreement with numerical simulation carried out in $[8,9]$, where was shown that erosion modulation occurs due to modulation of shielding layer.

Distance along the target [mm]

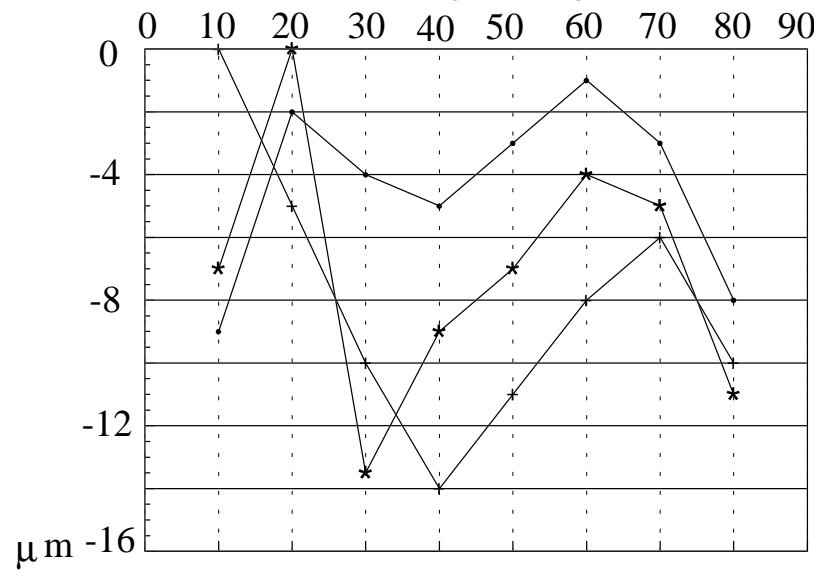

Figure 7 . Erosion profiles of quartz surface $(90 \times 90 \mathrm{~mm})$ for different cross-sections. Inclined incidence of plasma.

For the analysis of melt layer erosion copper, stainless steel and titanium were chosen. These materials were used for simplification of the first experiments and comparison with numerical simulation [9]. The main experiments were carried out in the third operation regime. Pictures of a $\mathrm{Cu}$ surface, taken with different magnification, are shown in Figs. 8 (a and b) for different doses of irradiation. One can see in Fig. $8 \mathrm{a}$ (irradiation with one pulse) that the border of the melt surface is degraded. On the right hand side there are small white impregnations. They are a mixture of micro-droplets, sputtered from the molten zone, and small zones beginning to melt. After 15 shots (Fig. 8b) the molten surface shows a wave structure shape. Besides this, pores of up to $30 \mu \mathrm{m}$ in diameter are seen. Obviously, these are tracks of micro-jets and levitation of bubbles. Apart from the molten zone one can see droplets of major size (up to $200 \mu \mathrm{m}$ ). A few such droplets are on the surface of the molten region.
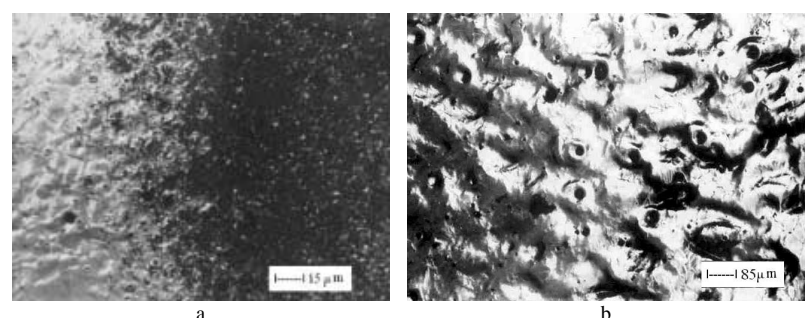

Figure 8. The pictures of $\mathrm{Cu}$ surfaces irradiated with plasma streams of $2 \mathrm{MW} / \mathrm{cm}^{2}$. a-1 shot; b-15 shots.

The weight losses of $\mathrm{Cu}$ and $\mathrm{Al}$ samples, normalized by their initial weight, are shown in Fig. 9 in function of the number of shots. The average value (by 15 shots) of losses is $3.83 .10^{-2} \mathrm{mg} / \mathrm{cm}^{2}$.pulse for $\mathrm{Cu}$ and $2.75 .10^{-1} \mathrm{mg} / \mathrm{cm}^{2}$.pulse for $\mathrm{Al}$.

Target mass, a.u.

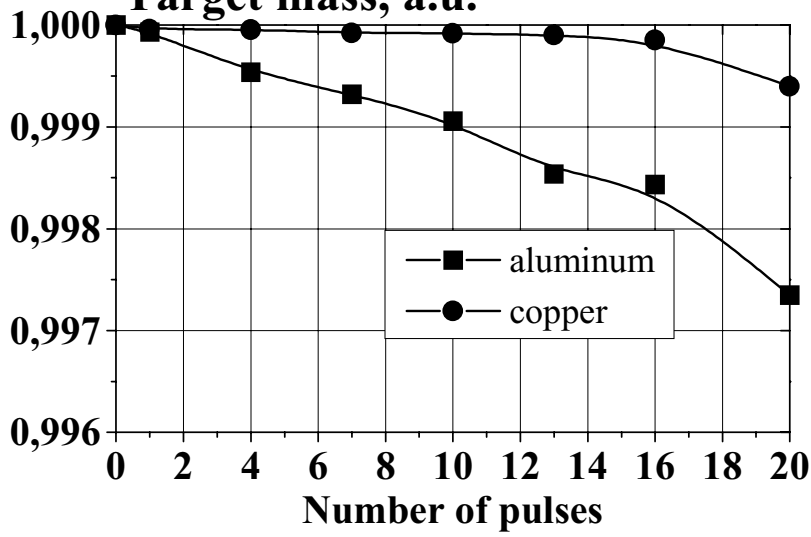

Figure 9. Variation of relative mass of irradiated samples with number of shots.

\section{Conclusion and discussion}

An investigation of plasma streams (with power density 2-20 MW and time duration of up to $0.35 \mathrm{~ms}$ ) interaction with magnetic field (up to $1.0 \mathrm{~T}$ ) was carried out. It was shown that the efficiency of plasma penetration through the mirror magnetic field is in strict dependence on plasma parameters and distance from the accelerator output to the mirror position. The latter result is explained by the geometry of the plasma stream outside the accelerator. Plasma streams are compressed at the distance of about $25 \mathrm{~cm}$ from the accelerator output. The length of compression region is $25-30 \mathrm{~cm}$. At the longer distances plasma streams diverge. The angle of divergence depends on plasma stream parameters. Therefore, plasma reflection by magnetic mirror is smaller for closer position of magnetic solenoid and smaller plasma divergence (for higher plasma density and velocity). The efficiency of plasma stream penetration through the magnetic mirror, being of an order (50-60)\% for high density plasma and magnetic solenoid position closer to plasma compression region, fell down 
to $(5-10) \%$ for smaller plasma density (the 2-d regime of QSPA operation) and longer distances from accelerator output.

Conditions for plasma magnetization, when propagating along the magnetic solenoid were found. It is obvious that plasma magnetizing efficiency should be in agreement with its parameters in magnetic field. Average $\beta$ value, being equal (30-40)\% for dense plasma in relatively weak magnetic field $\left(B_{Z}=0.36 \mathrm{~T}\right)$, fell down to (10-15)\% for plasmas of relatively low density.

Most of the experimental results on plasma-surface interaction were compared with a numerical code [9]. The main target settings of numerical simulation are as follows. 1) Plasma streams interact with the target and shielding plasma layer and due to this interaction plasma streams are decelerated. Transfer of power to the surface is due to the thermo-conductivity. 2) A two-dimensional MHD code is used simultaneously with Maxwell equations. Plasma radiation as well as plasma flow along and around the target is taken into account. 3) The losses in shield plasma layer, caused by ionization, radiation and magnetic field generation, are taken into account. 4) As to the target, the processes of thermo-conductivity, heat capacity, melting (erosion) and evaporation are taken into account in the code. 5) The task is accomplished as a time dependent, and real (experimental) profiles of power $P(t)$ are used. 6) From the solution of the equations we obtain $n(r, z, t)$ and $T(r, z, t)$, profiles of erosion, power radiation from the shield layer.

Some results of comparisons of experimental and numerical simulation data are shown in Table 2. Additionally, it was shown in experiments and calculations that the plasma density in the shielding layer was at least one order of magnitude higher as compared to the incident plasma one. It was confirmed a wave structure of the erosion profile of inclined quartz surface irradiated by plasma. The measured wavelength (around 6 $\mathrm{cm}$ ) was comparable to the one found in calculations. On the base of these recent investigations on plasmasurface interaction, further research will be carried out with more appropriate materials for fusion reactors.

Table 2. Comparison of numerical simulation and experimental results

\begin{tabular}{|c|c|c|c|c|}
\hline Material & Magnetic & $\begin{array}{c}\text { Target } \\
\text { inclination }\end{array}$ & Experiment & $\begin{array}{c}\text { Numerical } \\
\text { simulation }\end{array}$ \\
\hline Graphite erosion & 0 & $90^{\circ}$ & $2 \mu \mathrm{m} / \mathrm{pulse}$ & $1.6 \mu \mathrm{m} / \mathrm{pulse}$ \\
\hline Graphite erosion & $0.72 \mathrm{~T}$ & $90^{\circ}$ & $0.35 \mu \mathrm{m} / \mathrm{kJ}$ & $0.25 \mu \mathrm{m} / \mathrm{kJ}$ \\
\hline Quartz erosion & $0.72 \mathrm{~T}$ & $20^{\circ}$ & $7 \mu \mathrm{m} / \mathrm{pulse}$ & $6.5 \mu \mathrm{m} / \mathrm{pulse}$ \\
\hline Copper erosion & $0.72 \mathrm{~T}$ & $90^{\circ}$ & $\begin{array}{c}3.83 \times 10^{-2} \\
\mathrm{mg} / \mathrm{cm}^{2} \mathrm{pulse}\end{array}$ & - \\
\hline Aluminium erosion & $0.72 \mathrm{~T}$ & $90^{\circ}$ & $\begin{array}{r}2.75 \times 10^{-1} \\
\mathrm{mg} / \mathrm{cm}^{2} \mathrm{pulse}\end{array}$ & - \\
\hline
\end{tabular}

\section{References}

[1] A.I. Morozov, Nucl. Fusion Suppl., 111 (1969).

[2] A.K. Vinogradova, and A.I.Morozov, in: "Physics and Application of Plasma Accelerators" (Minsk: Nauka I Technika) 103 (1974).

[3] A.I. Morozov, Sov. J. Plasma Phys. 16, 69 (1990).

[4] A.I. Morozov, O.A. Shcurov, O.S. Pavlichenko, V.I. Tereshin, V.V. Chebotarev, Ya.F. Volkov, V.I. Kovalenko, N.V. Kulik, V.S. Manojlo, V.V. Marinin, D.G. Solyakov, V.V. Stal'tsov, and Yu.I. Tashchev, Plasma Devices and Operations 2, 155 (1992).

[5] V.I. Tereshin, Plasma Phys. Control. Fusion 37, 177 (1995).

[6] V.I. Tereshin, V.V. Chebotarev, I.E. Garkusha, V.A. Makhlaj, N.I. Mitina, A.I. Morozov, D.G. Solyakov, S.A.
Trubchaninov, A.V. Tsarenko, and H. Wuerz, Transactions of Fusion Technology 35, 248 (1999).

[7] V.V. Chebotarev, I.E. Garkusha, V.A. Makhlaj, N.I. Mitina, D.G. Solyakov, V.I. Tereshin, S.A. Trubchaninov, A.V. Tsarenko, and H. Wuerz, J. Nucl. Mater. 233237, 736 (1996).

[8] S. Pestchanyi, H. Wuerz, B. Bazylev, et al., [in:] Proc. 24-th EPS Conf. On Controlled Fusion and Plasma Physics, Berchtesgaden, June 9-13, 1997, 3, 981-984.

[9] H. Wuerz, S. Pestchanyi, I. Landman, V. Safronov, F. Kappler, B. Basylev, V. Tolkach, A. Burdakov, V. Koidan, D. Solyakov, V. Tereshin, Annual report of the Forschungszentrum Karlsruhe FZKA 6198, 1- 197 (1999). 